\title{
Identification of crucial genes involved in pathogenesis of regional weakening of the aortic wall
}

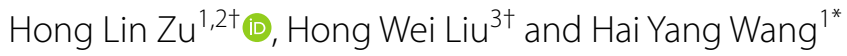

\begin{abstract}
Background: The diameter of the abdominal aortic aneurysm (AAA) is the most commonly used parameter for the prediction of occurrence of AAA rupture. However, the most vulnerable region of the aortic wall may be different from the most dilated region of AAA under pressure. The present study is the first to use weighted gene coexpression network analysis (WGCNA) to detect the coexpressed genes that result in regional weakening of the aortic wall.

Methods: The GSE165470 raw microarray dataset was used in the present study. Differentially expressed genes (DEGs) were filtered using the "limma" R package. DEGs were assessed by Gene Ontology biological process (GO-BP) and Kyoto Encyclopedia of Genes and Genomes (KEGG) analyses. WGCNA was used to construct the coexpression networks in the samples with regional weakening of the AAA wall and in the control group to detect the gene modules. The hub genes were defined in the significant functional modules, and a hub differentially expressed gene (hDEG) coexpression network was constructed with the highest confidence based on protein-protein interactions (PPIs). Molecular compound detection (MCODE) was used to identify crucial genes in the hDEG coexpression network. Crucial genes in the hDEG coexpression network were validated using the GSE7084 and GSE57691 microarray gene expression datasets.
\end{abstract}

Result: A total of 350 DEGs were identified, including 62 upregulated and 288 downregulated DEGs. The pathways were involved in immune responses, vascular smooth muscle contraction and cell-matrix adhesion of DEGs in the samples with regional weakening in AAA. Antiquewhite 3 was the most significant module and was used to identify downregulated hDEGs based on the result of the most significant modules negatively related to the trait of weakened aneurysm walls. Seven crucial genes were identified and validated: ACTG2, CALD1, LMOD1, MYH11, MYL9, MYLK, and TPM2. These crucial genes were associated with the mechanisms of AAA progression.

Conclusion: We identified crucial genes that may play a significant role in weakening of the AAA wall and may be potential targets for medical therapies and diagnostic biomarkers. Further studies are required to more comprehensively elucidate the functions of crucial genes in the pathogenesis of regional weakening in AAA.

Keywords: Abdominal aortic aneurysm, Differentially expressed genes, Weighted gene coexpression network analysis, Crucial genes, Vascular smooth muscle cells

\footnotetext{
*Correspondence: wanghaiyang@hrbmu.edu.cn

${ }^{\dagger}$ Hong Lin Zu and Hong Wei Liu contributed equally to this work. ${ }^{1}$ Department of Vascular Interventional Surgery, the First Affiliated Hospital of Harbin Medical University, No.23 Youzheng Str, Nangang District, Harbin 150001, Heilongjiang, China

Full list of author information is available at the end of the article
}

\section{Introduction}

Ruptured abdominal aortic aneurysm (AAA) is an important complication caused by AAA, resulting in severe haemorrhagic shock, and is associated with a mortality rate as high as $81 \%$ [1]. A considerable part of patients with ruptured AAA (rAAA) die before admission to the 
hospital. Most patients with AAA are asymptomatic, and population-based screening studies have reported the prevalence rates of AAA ranging from $1.6 \%$ to $7.2 \%$ of the general population from 60 to 65 years of age or older [2]. Smoking, uncontrolled blood pressure, older age, female sex and aneurysm diameter are risk factors for AAA rupture. In current clinical practice, the diameter of the aneurysm is the most commonly used parameter for the prediction of occurrence of AAA rupture, and patients with larger diameter aneurysms are at the highest risk of aortic rupture. The annual rupture rate is approximately $1 \%$ in patients with aneurysms $40-49 \mathrm{~mm}$ in diameter and $11 \%$ for patients with aneurysms $50-59 \mathrm{~mm}$ in diameter [2]. Elective surgical treatment includes open surgical repair or endovascular aneurysm repair provided to the majority of diagnosed patients at low or acceptable surgical risk with large AAAs $\geq 55 \mathrm{~mm}$ in diameter in men and $\geq 50 \mathrm{~mm}$ in diameter in women [3]. Despite a possibility of a rupture, regular follow-up is preferentially recommended versus surgical intervention in the case of small AAAs [4]; thus, specific drug therapy to limit the growth or rupture of AAAs is unavailable.

Molecular and histopathological evaluation of tissue samples of abdominal aorta provides a comprehensive approach to identify broadly coordinated gene expression in biological pathways involved in rAAA pathobiology. E Choke et al. [5] used the whole transcriptome approach to confirm overexpression of the genes $I L-8$, $I L-6, P T G S 2, P R O K 2$ and SELE involved in the immune and inflammatory responses at the AAA rupture sites. Gäbel et al. [6] utilized aortic wall tissue specimens for investigation of differentially expressed genes (DEGs) and validated that HILPDA, ANGPTL4, LOX, SRPX2, FCGBP, ADAMTS9, STC1, GFPT2, GAL3ST4 and CCL4L1 were overexpressed candidate genes related to angiogenesis, adipogenesis, epithelial-mesenchymal transition and the HIF-1a pathway apparently pivotal for AAA progression towards rupture. Based on publicly available original datasets, Lei et al. [7] performed weighted gene coexpression network analysis (WGCNA) to identify DEGs and infiltrated immune cells, including naïve $B$ cells and both resting and activated $\mathrm{CD} 4^{+}$memory $\mathrm{T}$ cells, which are significantly upregulated in ruptured AAA. WGCNA was used to explore the system-level functionality of the genes [8]. Previous studies used WGCNA with microarray data to identify the hub genes in AAA that may act as potential targets for medical therapy and as diagnostic biomarkers $[9,10]$.

Recently, Vivian et al. [11] suggested that the most dilated regions of AAA are not the most vulnerable regions under pressure and are ruptured due to the presence of a weakened region in the aortic wall. Forneris et al. [12] defined the regional aortic weakness (RAW) index as the sum of time-averaged wall shear stress, intraluminal thrombus and large strain scores for each patch of the aneurysm, and the RAW index values $>6$ according to the ultimate tensile strength (UTS) values indicate a weak wall. This approach will assist in improving clinical decision-making in the management of aortic pathology. In the current study, WGCNA was constructed using the high RAW index and control samples to identify crucial genes in the constructed gene modules that were involved in regional weakening of the aortic wall. These genes may provide evidence for identification of biological mechanisms of pathogenesis in rAAA.

\section{Materials and methods Data collection}

The raw data of the GSE165470 [12], GSE7084 [13] and GSE57691 microarrays were acquired from the Gene Expression Omnibus (GEO) database (https://www. ncbi.nlm.nih.gov/geo/); the data were uploaded by the University of Calgary, Wayne State University School of Medicine and James Cook University. We used publicly accessible data and did not conduct human or animal experiments; hence, Institutional Ethics Committee approval was not required.

\section{DEG screening and functional annotation analysis}

The "Limma" $R$ package was used to evaluate DEGs of the GEO chip data. The DEG cut-off criteria were set as $P<0.05$ and $|\log 2 \mathrm{FC}|>2$, and the "ggplot" package was used to generate the volcano map. Upregulated and downregulated DEGs were used for Gene Ontology biological process (GO-BP) and Kyoto Encyclopedia of Genes and Genomes (KEGG) analyses by using the Database for Annotation, Visualization and Integrated Discovery (DAVID) v6.8 (https://david.ncifcrf.gov/) [14]. The top 10 statistically significant GO-BP and KEGG pathway terms for DEGs were recorded. The levels of enriched genes $\geq 2$ and $P<0.05$ were considered statistically significant differences.

\section{Construction of a weighted gene coexpression network and hub gene screening}

The "WGCNA" R package was applied to generate a coexpression network to explore the clustered functionality of the genes and identify the core regulatory genes that were biologically meaningful. All genes were involved in the analysis. The absolute value of the Pearson correlation was calculated, and the raw gene matrix was converted into a similarity expression matrix. A suitable soft threshold $(\beta)$ was selected to construct a network that accounted for the scale-free characteristics, and the linear regression model fitting index R2 was quantified to assess the scale-free topology of the network. Then, the 
weighted adjacency matrix was generated and transformed into a topological overlap matrix (TOM). The TOM-based dissimilarity measure in average linkage hierarchical clustering was used for functional module detection. Similar dynamic modules were merged based on the height cut-off of the dendrogram at the minimum module of 30. Highly similar dynamic modules were further merged at a threshold of 0.2.

\section{Construction of the DEG coexpression network}

Module membership and gene significance were calculated to validate significant functional modules related to clinical traits. The hub genes were defined as the genes with the module membership $>0.8$ in significant functional modules. Subsequently, a list of DEGs was overlapped with a list of the hub genes to construct a coexpression network of the hubs of differentially expressed genes (hDEGs).

\section{Construction of a PPI network and identification of crucial genes}

The Search Tool for the Retrieval of Interacting Genes (STRING) database version 11.0 (string-db.org) was used to search for protein-protein interactions (PPI) with the highest confidence (interaction score $>0.9$ ) to determine the interconnections of hDEGs in the functional modules [15]. Molecular complex detection (MCODE), a plug-in of Cytoscape 3.7.1, was used to detect and visualize the hDEG clusters, and hDEGs in the most significant clusters were considered crucial genes. Finally, crucial genes were validated based on the mRNA expression levels in the GSE7084 and GSE57691 raw microarray datasets by comparison between the AAA and control groups.

\section{Statistical analysis}

R 4.1 was used for data preprocessing, DEG screening and WGCNA. DAVID was used for functional annotation analysis, and the MCODE plug-in of Cytoscape v3.7.1 was utilized to identify crucial genes. The criteria for these bioinformatic analyses are described in the corresponding subsections. Unpaired Student's $t$-test was used to analyse the mRNA expression levels and compare the AAA and control groups. Statistical analysis was performed using GraphPad Prism v.6.01. $P<0.05$ was considered significant.

\section{Results}

\section{Data characteristics}

The GSE165470 datasets included a total of two groups of 10 AAA patch samples. According to the RAW index (RAW >6), 6 samples and 4 samples were assigned to the disease group and control group, respectively. The samples of the disease group had regional weakening of the AAA wall tissues. The gene expression matrix was acquired by transforming the probe ID matrix annotated with gene symbols from the GPL23126 platform data. The GSE7084 and GSE57691 microarray gene expression datasets contained 69 AAA tissues, and 21 controls were only used for validation of crucial genes. (Table 1).

\section{DEG screening}

A total of 350 DEGs were screened with a $P$-value $<0.05$ and a $\mid \log 2$-fold-change $\mid>2$; these DEGs contained 62 upregulated and 288 downregulated genes. The expression levels and volcano plot of DEGs are visualized in Fig. 1A, B. The top 10 DEGs with the highest up- and downregulated expression levels are shown in Table 2.

\section{Functional annotation and pathway enrichment analysis}

A total of 35 GO-BP terms were enriched in upregulated DEGs, and 77 GO-BP terms were enriched in downregulated DEGs; the immune response and cell adhesion were the terms most significantly enriched by up- and downregulated DEGs in GO-BP. Nine and seventeen KEGG pathways were enriched in up- and downregulated DEGs, respectively, and ECM-receptor interaction and focal adhesion were the most significantly enriched KEGG pathways. The top 10 GO-BP terms and KEGG pathways enriched by DEGs are shown in Fig. 2, and DEGs involved in each enrichment pathway are shown in the supplementary file (Data S1).

\section{The results of weighted gene coexpression network analysis}

The "WGCNA" $\mathrm{R}$ package was used to construct a coexpression network for the 6 and 4 samples of the disease and control groups, respectively. The histogram and linear plot indicated that a cut-off R2 value of 0.78 and soft threshold $\beta$ value of 18 should be used to build a coexpression scale-free topology network (Fig. 3A, B, C, D). Heatmap of the constructed gene coexpression network indicated the presence of several blocks with clear colour differences in the gene cluster tree (Fig. 3E). Nine highly similar dynamic modules were detected based on TOM matrices combined with cutting and merging of the dynamic dendrogram tree, and

Table 1 Description of the 3 GSE microarray datasets

\begin{tabular}{lllll}
\hline Series & Tissues & Disease & Control & platforms \\
\hline GSE165470 & $\begin{array}{l}\text { Abdominal Aortic } \\
\text { aneurysm patch }\end{array}$ & 6 & 4 & GPL23126 \\
GSE7084 & Abdominal Aorta & 10 & 11 & GPL2507 \\
GSE57691 & Abdominal Aorta & 59 & 10 & GPL10558 \\
\hline
\end{tabular}




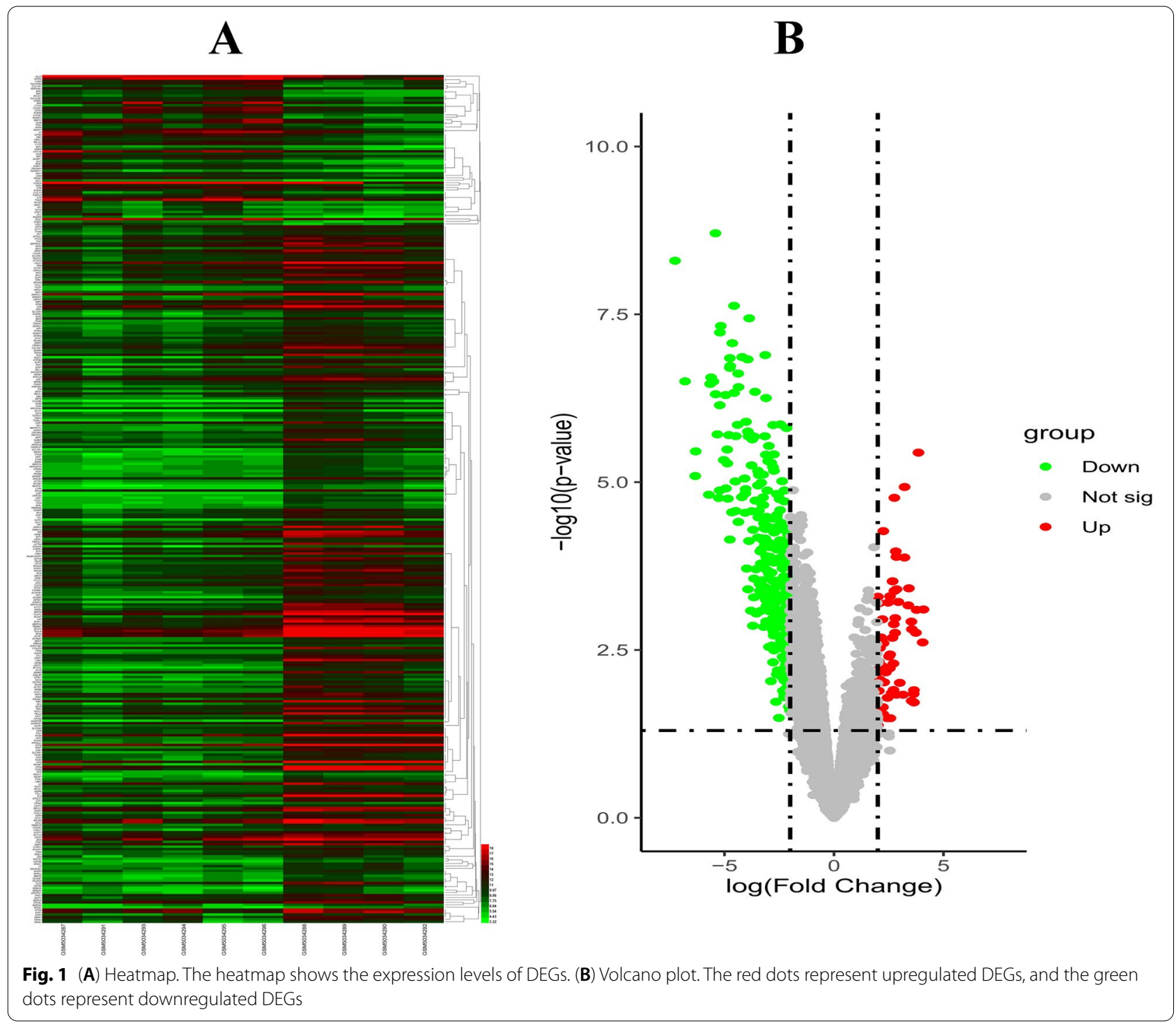

the antiquewhite 3 module had the most significant negative correlation with the trait of weakened aneurysm walls (Fig. 3F, G).

\section{Identification of hub DEGs}

The antiquewhite3 module included 3,912 genes (Fig. 4A). The 366 hub genes were identified by calculating the correlations to the antiquewhite 3 module and the trait of weakened aneurysm walls. Then, the hub genes were overlapped with DEGs, and 186 downregulated hDEGs were identified (Fig. 4B), which was in agreement with the results of the most significant module that was negatively related to the trait of weakened aneurysm walls.

\section{Construction of a hub DEG coexpression network and identification of crucial genes}

All hDEGs were the genes downregulated in the samples with weakened regional aneurysm wall versus the samples of the control group. It is unlikely that a single gene or pathway can cause regional weakening of the aneurysm walls. An hDEG PPI network with 55 nodes and 109 edges was constructed with the highest confidence (Fig. 5A). In addition, 3 interconnected cluster networks of hDEGs were identified and visualized by using MCODE (Fig. 5B, C, D). ACTA2, ACTG2, CALD1, LMOD1, MYH11, MYL9, MYLK, $T P M 1, T P M 2$ and $V C L$ were crucial genes with the highest interconnected cluster scores (Fig. 5B). Apart from ACTA2, $T P M 1$ and $V C L$, all other crucial genes in the hDEG coexpression network were successfully validated using the 
Table 2 Top 10 up- and downregulated DEGs

\begin{tabular}{|c|c|c|c|}
\hline Gene symbol & $\log F C$ & $P$-Value & Gene official full name \\
\hline \multicolumn{4}{|l|}{ UP-regulated } \\
\hline IGLL5 & 4.085 & 0.000788 & Immunoglobulin lambda like polypeptide 5 \\
\hline SFRP4 & 4.048333 & 0.00244 & Secreted frizzled related protein 4 \\
\hline SAA2 & 3.856667 & 3.63E-06 & Serum amyloid A2 \\
\hline HLA-DRB5 & 3.7725 & 0.000798 & Major histocompatibility complex, class II, DR beta 5 \\
\hline CD79A & 3.7375 & 0.00175 & CD79a molecule \\
\hline IBSP & 3.65 & 0.019102 & Integrin binding sialoprotein \\
\hline MMP12 & 3.648333 & 0.012571 & Matrix metallopeptidase 12 \\
\hline CXCL13 & 3.636667 & 0.014243 & C-X-C motif chemokine ligand 13 \\
\hline DCC & 3.555833 & 0.001552 & DCC netrin 1 receptor \\
\hline FDCSP & 3.548333 & 0.018089 & Follicular dendritic cell secreted protein \\
\hline \multicolumn{4}{|c|}{ DOWN-regulated } \\
\hline CNN1 & -7.26083 & 5.03E-09 & Calponin 1 \\
\hline ITGA8 & -6.8025 & $3.15 \mathrm{E}-07$ & Integrin subunit alpha 8 \\
\hline ACTC1 & -6.3375 & $8.10 \mathrm{E}-06$ & Actin, alpha, cardiac muscle 1 \\
\hline MYH11 & -6.31667 & $3.48 \mathrm{E}-06$ & Myosin heavy chain 11 \\
\hline MFAP4 & -5.71417 & $1.54 \mathrm{E}-05$ & Microfibrillar associated protein 4 \\
\hline$P L N$ & -5.65833 & $3.44 \mathrm{E}-07$ & Phospholamban \\
\hline MYOCD & -5.61333 & $2.76 \mathrm{E}-07$ & Myocardin \\
\hline PPP1R12B & -5.51 & 3.17E-07 & Protein phosphatase 1 regulatory subunit 12B \\
\hline RCAN2 & -5.41333 & 1.96E-09 & Regulator of calcineurin 2 \\
\hline $\mathrm{PCDH7}$ & -5.405 & 4.90E-07 & Protocadherin 7 \\
\hline
\end{tabular}

GSE7084 and GSE57691 microarray gene expression datasets (Fig. 6 and supplementary file Data S2).

\section{Discussion}

Genes in patients with ruptured AAA were likely to be more actively involved in inflammation, proteolysis, pathways regulating VSMC lineages and ECM homeostasis. In addition to the activation of immune responses, the AAA wall is characterized by proteolytic fragmentation of the extracellular matrix (ECM) and phenotypic modulation or apoptosis of vascular smooth muscle cells (VSMCs), which result in a thin and degraded media, few VSMCs and elastic components of the aneurysm walls $[16,17]$.

We identified 350 DEGs, including 62 upregulated and 288 downregulated genes, using the raw microarray data of GSE165470. The results of GO-BP and KEGG pathway enrichment analyses indicated that most of upregulated DEGs were involved in innate and adaptive immune responses, $\mathrm{B}$ cell activation and proliferation, positive regulation of angiogenesis, etc. In agreement with the results reported in human and animal models, pathological examination of AAA samples detected a wide array of innate and adaptive immune cells, such as mast cells, macrophages, neutrophils, dendritic cells, B cells and T cells, which infiltrated the aortic wall [18].
Inflammation and immunological responses play an important role in the progression of AAA. HLADRB5 encodes the heavy chain of human leukocyte antigen (HLA) II, which activates $\mathrm{T}$ helper cells to increase the inflammatory response. Marfan syndrome patients with progressive aortic root dilatation have increased expression of $H L A-D R B 5$, which indicates an increase in the inflammatory response corresponding to progressive aortic disease [19]. HLA II genes (HLA-DRB1*15, HLA$D R B 1 * 04$ and $H L A-D R B 1 * 02)$ have been identified as the factors involved in AAA development [20]. CXCL13 promotes the migration of $B$ lymphocytes and was validated as a novel inflammation gene linked to AAA based on increased gene expression profiles [21]. Kevin et al. [22] demonstrated that the media and adventitia layers of AAA contain higher levels of CXCL13, which triggers the recruitment of $B$ cells, resulting in a high presence of B cells in the adventitia layer. Simultaneously, phenotypic changes in VSMCs can act as lymphoid tissue organizer-like cells in inflammatory environment involved in human aortic lymphoid neogenesis and are subjected to persistent inflammation and immune responses.

Interestingly, most downregulated DEGs were involved in various biological pathways, such as vascular smooth muscle contraction, regulation of actin cytoskeleton, 

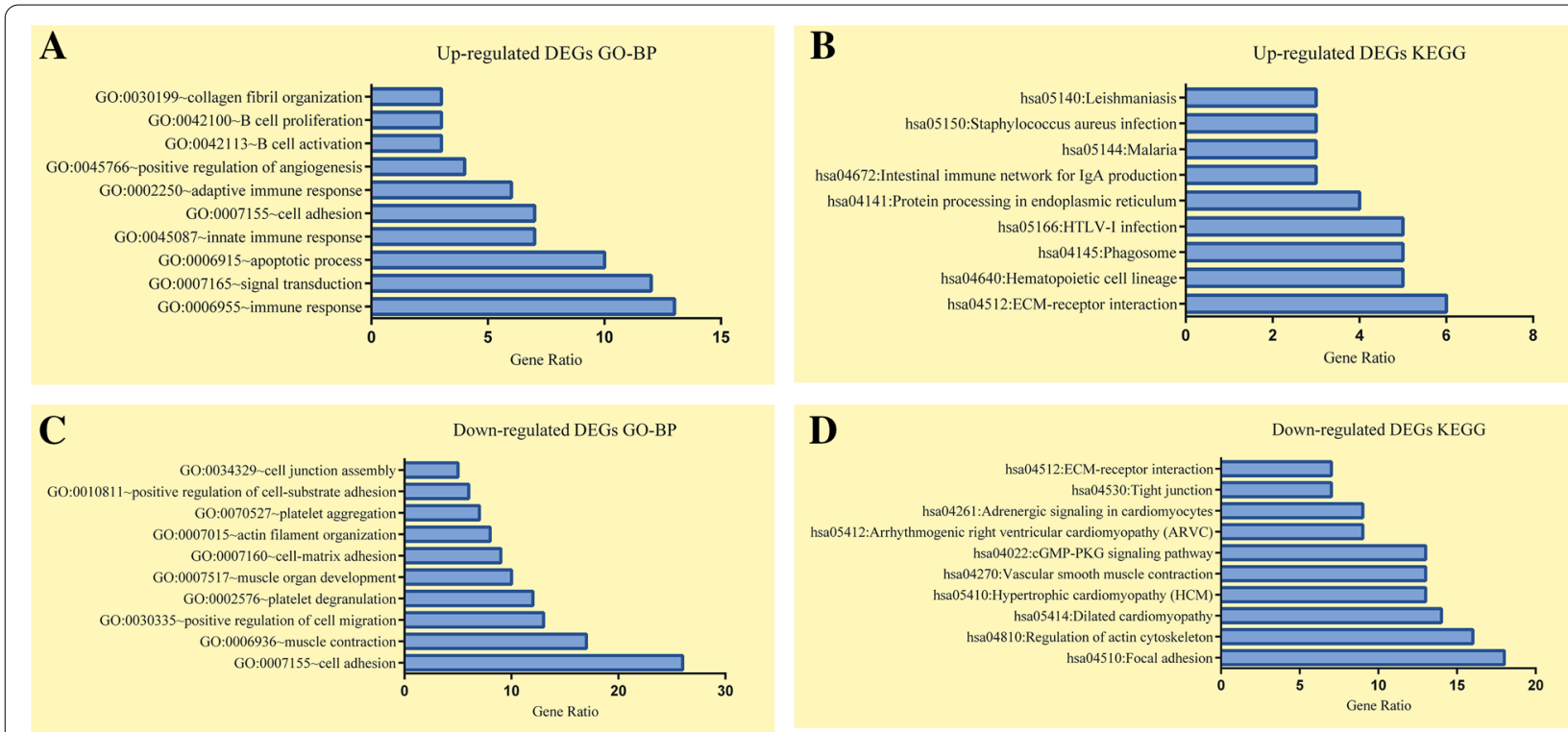

Fig. 2 Top 10 GO-BP terms and KEGG pathways enriched in DEGs. (A, B) Functional enrichment analyses of the top 10 GO-BP and KEGG pathways enriched by upregulated DEGs according to the gene ratio values and in ascending order of the P-value. (C, D) The same criteria were applied for enrichment analysis of downregulated DEGs. The length of the histogram represents the number of the genes. Details of the GO-BP and KEGG pathway terms are described on the left Y-axis

cell-matrix adhesion and ECM-receptor interaction. ITGA8 was expressed at a high level in aortic SMC tissue of human origin with contractile phenotype; moreover, ectopic MYOCD expression resulted in a consistent increase in ITGA8 mRNA levels in rat, mouse and human SMCs [23]. Moreover, ITGA8 may be an assembly that regulates normal SMC differentiation, and decreased IGTA8 expression leads to impaired VSMC contractility [24]. MFAP4 is an ECM protein expressed at a high level in human elastic tissues specifically localized in elastic fibres in the blood vessels [25]. Lindholt et al. [26] used immunohistochemistry to demonstrate a higher MFAP4 staining in healthy tissues than that in AAA tissues, and high levels of plasma MFAP4 are significantly negatively correlated with the aorta growth rate, resulting in decreased surgical repair in AAA.

However, monogenic disorders are rarely associated with AAA progression. The present study is the first to construct a coexpression network for both high and low RAW index samples by using the WGCNA algorithm to detect the coexpressed genes resulting in weakening of the regional aortic wall. Seven crucial genes, ACTG2, CALD1, LMOD1, MYH11, MYL9, MYLK, and TPM2, were identified and validated; moreover, these coexpressed genes may play an important role in regional weakening the aortic wall.

ACTG2 belong to the actin family and is highly conserved cytoskeletal proteins. ACTG2 encodes a protein specific to visceral smooth muscle tissue [27]. MYH11 encodes a smooth muscle myosin belonging to the myosin heavy chain family. Utako et al. [28] demonstrated that circulating plasma MYH11 levels were significantly higher in AAA. MYLK encodes myosin light chain kinase, which phosphorylates myosin regulatory light chains to facilitate myosin interaction with actin filaments to produce contractile activity, and diminished levels of MYL9 cause a failure in actomyosin-based contraction [29].

The major function of VSMC contractile force requires cyclic interactions between SMC a-actin and the $\beta$-myosin heavy chain encoded by MYH11 [27]. MYH11 and MYLK gene mutation-induced switching of aortic SMCs from a contractile to a synthetic inflammatory phenotype is accompanied by secretion of abundant MMPs, inflammatory cytokines and chemokines and growth factors and by production of reactive oxygen species (ROS) and proteoglycans in the aortic adventitia to create a vicious cycle leading to the degradation of ECM and weakening of the aortic wall, resulting in aortic rupture [30-32]. Shalata et al. [33] reported that the MYLK Ala1491Ser mutation affected kinase activity and clinically presented with vascular aneurysm and dissection in thoracic and/or abdominal aorta and peripheral or visceral arteries.

The $L M O D 1$ and $C A L D 1$ were implicated in differentiation-adapted phenotype of smooth muscle cells induced by serum response factor (SRF)/myocardin (MYOCD) transcription factors [34, 35]. Knockdown of LMOD1 

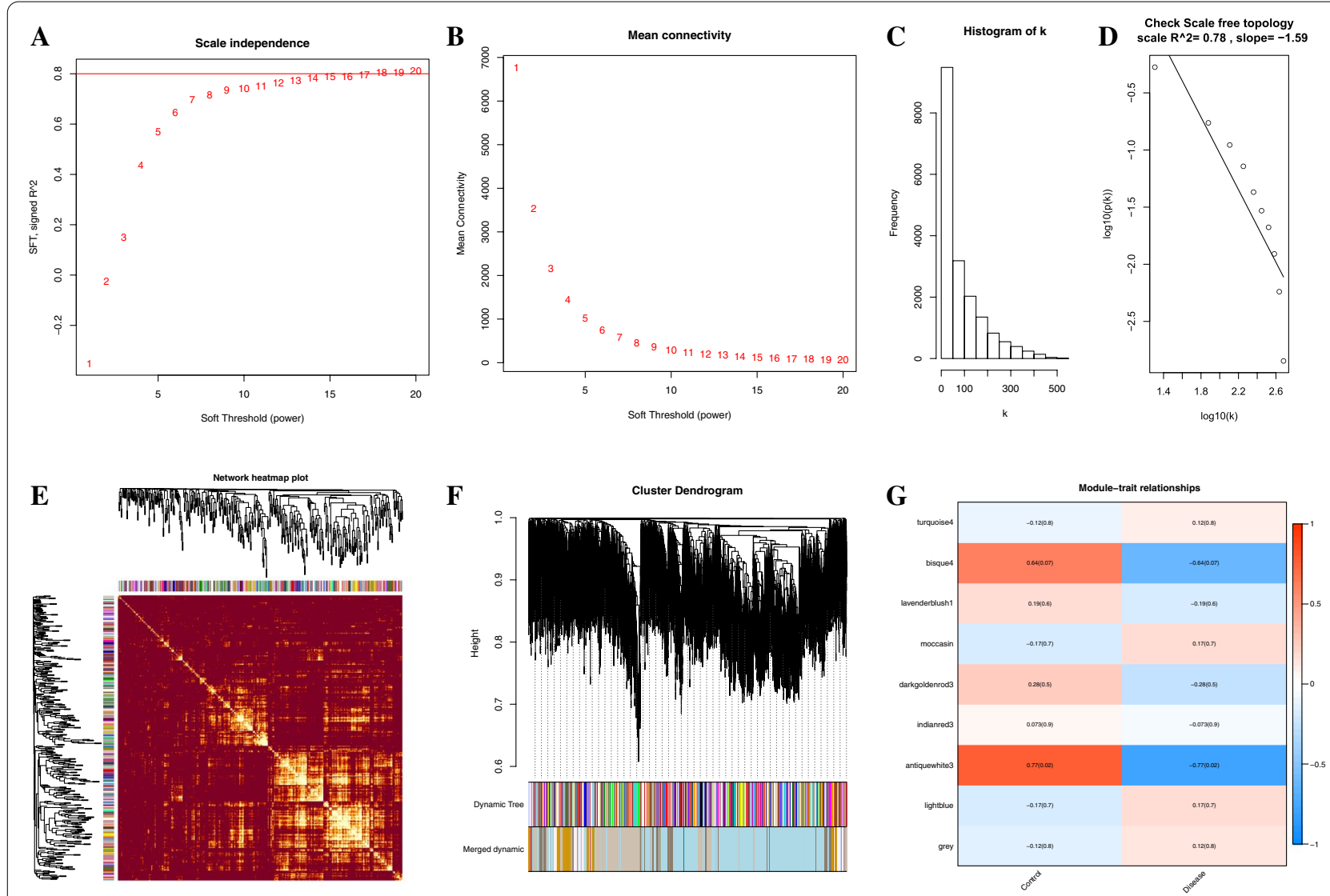

Fig. 3 Algorithm of WGCNA. (A, B) An optimal soft-threshold power was selected to construct a scale-free topology network. (C, D) Scale-free topology with a soft threshold $\beta$ value of 18. (E) Heatmap illustrating the TOM of the gene cluster tree; light colours represent a higher number of connections. (F) Module detection was based on hierarchical cluster analysis with cut-off and merging of the dendrogram, and the corresponding modules with various colours were obtained. (G) Calculated correlation coefficients between the modules and clinical traits. The antiquewhite3 module had the most negative correlation with the disease group

resulted in increased proliferation and migration and decreased cell contraction of human coronary artery SMCs [36], and CALD1 was significantly expressed at low levels in VSMCs from carotid artery plaques, which were identified as critically involved in the development and progression of atherosclerosis [37]. TPM2 encodes beta-tropomyosin, a subtype of tropomyosin that belongs to the family of actin filament-binding proteins [38].
A

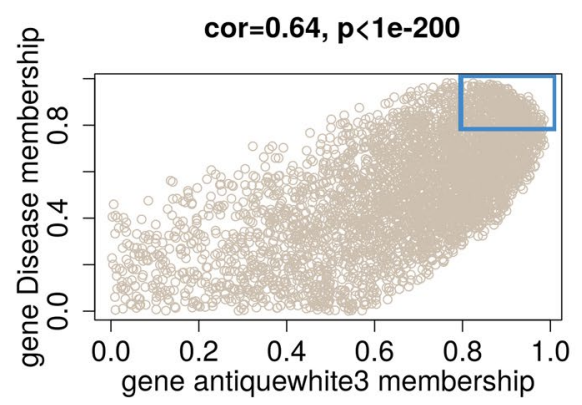

B

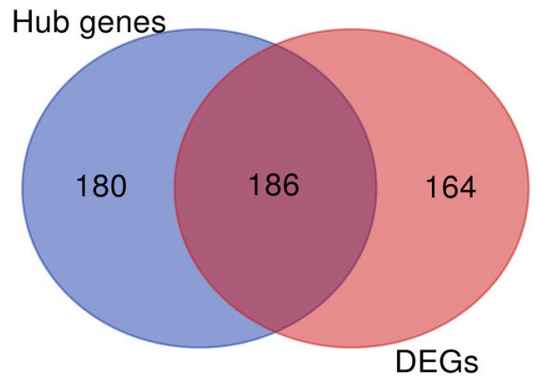

Fig. 4 Algorithm of identification of the hub DEGs. (A) Gene sets highly related to the antiquewhite3 module and disease trait with a Pearson correlation coefficient of 0.64 and $P<0.05$. Hub genes were identified with module membership $>0.8$ in the blue rectangle. (B) A total of 186 downregulated hDEGs were obtained by overlapping 367 hub genes and 350 DEGs 

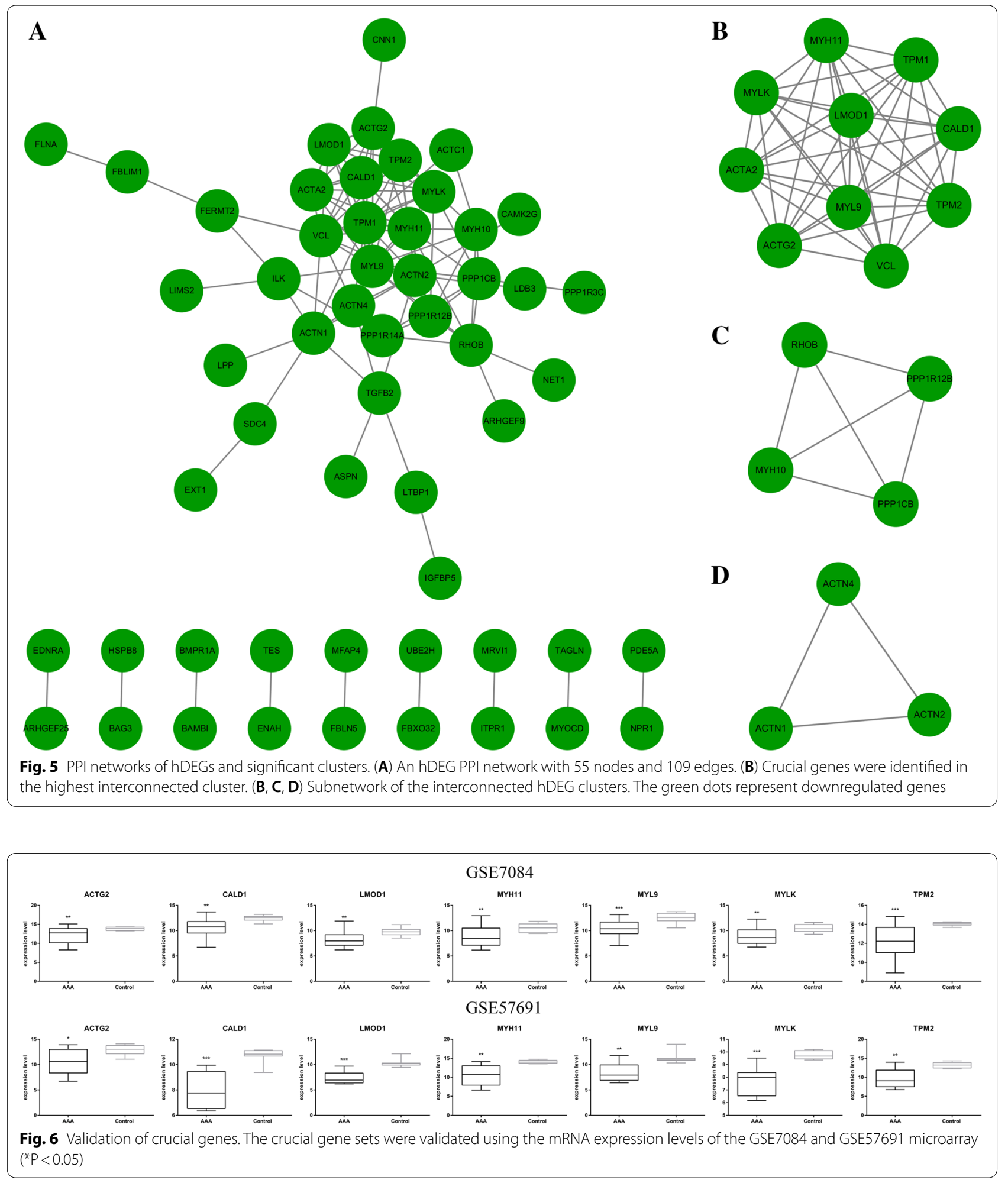

Downregulation of TPM2 may lead to disorders in the formation and movement of VSMCs in the development of atherosclerosis [39].
In conclusion, hDEGs are involved in phenotypic changes and even apoptosis of VSMCs, and degraded ECM results in regional weakening of the aortic wall. 
Consequent biomechanical stress exerted on the wall dilates the aneurysm that ruptures when the AAA wall stress exceeds the tensile strength of the wall. We identified crucial genes that play a notable role in regional weakening of the AAA wall. The present study provides a reference for future studies, elucidating detailed biological mechanisms of crucial genes in pathogenesis of rAAA.

\section{Abbreviations}

AAA: Abdominal aortic aneurysm; rAAA: Ruptured abdominal aortic aneurysm; RAW: Regional aortic weakness; UTS: Ultimate tensile strength; GEO: Gene Expression Omnibus; DEGs: Differentially expressed genes; WGCNA: Weighted gene coexpression network analysis; GO: Gene Ontology; BP: Biological process; KEGG: Kyoto Encyclopedia of Genes and Genomes; DAVID: Database for Annotation, Visualization and Integrated Discovery; TOM: Topological overlap matrix; STRING: Search Tool for the Retrieval of Interacting Genes; PPI: Protein-protein interaction; MCODE: Molecular Complex Detection; hDEGs: Hub differentially expressed genes.
\end{abstract}

\section{Supplementary Information}

The online version contains supplementary material available at https://doi. org/10.1186/s41065-021-00200-1.

\section{Additional file 1.}

Additional file 2.

\section{Acknowledgements}

The raw data of the GSE165470, GSE7084 and GSE57691 microarrays were acquired from the Gene Expression Omnibus (GEO) database; the data were uploaded by the University of Calgary, Wayne State University School of Medicine and James Cook University.

\section{Authors' contributions}

Conception and design: $\mathrm{Zu} \mathrm{HL}$; Data process, visualization, analysis, and interpretation: Zu HL, Wang HY and Liu HW. Manuscript writing: Zu HL and Liu HW. The author(s) read and approved the final manuscript.

\section{Funding}

This research was funded by the National Natural Science Foundation of China, grant number 81570424.

\section{Availability of data and materials}

The GSE datasets are available in the GEO database (https://www.ncbi.nlm.nih. gov/geo/).

\section{Declarations}

Ethics approval and consent to participate

Not applicable.

\section{Consent for publication}

All authors have read and approved the final manuscript.

\section{Competing interests}

All authors declare there are no competing interests.

\section{Author details}

1 'Department of Vascular Interventional Surgery, the First Affiliated Hospital of Harbin Medical University, No.23 Youzheng Str, Nangang District, Harbin 150001, Heilongjiang, China. ${ }^{2}$ Department of Vascular Surgery, Tian Jin First Center Hospital, Tianjin 300192, China. ${ }^{3}$ Department of Vascular Surgery, the Second Affiliated Hospital of Harbin Medical University, Harbin 150086, Heilongjiang, China.

Received: 10 July 2021 Accepted: 1 September 2021

Published online: 02 December 2021

\section{References}

1. Reimerink JJ, van der Laan MJ, Koelemay MJ, Balm R, Legemate DA. Systematic review and meta-analysis of population-based mortality from ruptured abdominal aortic aneurysm. Br J Surg. 2013;100:1405-13. https://doi.org/10.1002/bjs.9235.

2. Guirguis-Blake JM, Beil TL, Senger CA, Coppola EL. Primary Care Screening for Abdominal Aortic Aneurysm: Updated Evidence Report and Systematic Review for the US Preventive Services Task Force. JAMA. 2019;322:2219-38. https://doi.org/10.1001/jama.2019.17021.

3. Chaikof EL, Dalman RL, Eskandari MK, Jackson BM, Lee WA, Mansour MA et al. The Society for Vascular Surgery practice guidelines on the care of patients with an abdominal aortic aneurysm. J Vasc Surg. 2018;67:2-77. https://doi.org/10.1016/j.jvs.2017.10.044

4. Galyfos G, Sigala F, Mpananis K, Vouros D, Kimpizi D, Theodoropoulos C, et al. Small abdominal aortic aneurysms: Has anything changed so far? Trends Cardiovasc Med. 2020;30:500-4. https://doi.org/10.1016/.tcm. 2019.11.006.

5. Choke E, Cockerill GW, Laing K, Dawson J, Wilson WRW, Loftus IM, et al. Whole Genome-expression Profiling Reveals a Role for Immune and Inflammatory Response in Abdominal Aortic Aneurysm Rupture. Eur J Vasc Endovasc Surg. 2009;37:305-10. https://doi.org/10.1016/j.ejvs.2008. 11.017 .

6. Gäbel G, Northoff BH, Weinzierl I, Ludwig S, Hinterseher I, Wilfert W, et al. Molecular Fingerprint for Terminal Abdominal Aortic Aneurysm Disease. J Am Heart Assoc. 2017:6:e006798. https://doi.org/10.1161/JAHA.117. 006798.

7. Lei C, Yang D, Chen S, Chen W, Sun X, Xiao Wu, et al. Patterns of immune infiltration in stable and raptured abdominal aortic aneurysms: A geneexpression-based retrospective study. Gene. 2020;762: 145056. https:// doi.org/10.1016/j.gene.2020.145056.

8. Bin Zhang, Steve Horvath. A General Framework for Weighted Gene CoExpression Network Analysis. Stat Appl Genet Mol Biol. 2005;4:Article17. https://doi.org/10.2202/1544-6115.1128

9. Chen S, Yang D, Lei C, Li Y, Sun X, Chen M, et al. Identification of crucial genes in abdominal aortic aneurysm by WGCNA. Peer J. 2019;7: e7873. https://doi.org/10.7717/peerj.7873.

10. Chen S, Yang D, Liu B, Chen Y, Ye We, Chen M, et al. Identification of crucial genes mediating abdominal aortic aneurysm pathogenesis based on gene expression profiling of perivascular adipose tissue by WGCNA. Ann Transl Med. 2021;9:52. https://doi.org/10.21037/atm-20-3758.

11. Vivian Carla Gomes, Madhavan Lakshmi Raghavan, Luiz Fernando Ferraz da Silva, Jorge Gomes, Gina Camillo Silvestre, Alexandre Queiroz, et al. Experimental Study of Rupture Pressure and Elasticity of Abdominal Aortic Aneurysms Found at Autopsy. Ann Vasc Surg. 2021;70:517-527. https://doi.org/10.1016/j.avsg.2020.08.111

12. Arianna Forneris, Jacob Kennard, Alina Ismaguilova, Robert D Shepherd, Deborah Studer, Amy Bromley, et al. Linking Aortic Mechanical Properties, Gene Expression and Microstructure: A New Perspective on Regional Weakening in Abdominal Aortic Aneurysms. Front Cardiovasc Med. 2021;8:631790. https://doi.org/10.3389/fcvm.2021.631790

13. Pahl MC, Erdman R, Kuivaniemi H, Lillvis JH, Elmore JR, Tromp G. Transcriptional (ChIP-Chip) Analysis of ELF1, ETS2, RUNX1 and STAT5 in Human Abdominal Aortic Aneurysm. Int J Mol Sci. 2015;16:11229-58. https://doi. org/10.3390/ijms160511229.

14. Huang DW, Sherman BT, Lempicki RA. Systematic and integrative analysis of large gene lists using DAVID Bioinformatics Resources. Nature Protoc. 2009;4:44-57. https://doi.org/10.1038/nprot.2008.211.

15. Damian Szklarczyk, Annika L Gable, David Lyon, Alexander Junge, Stefan Wyder, Jaime Huerta-Cepas, et al. STRING v11: protein-protein association networks with increased coverage, supporting functional discovery in genome-wide experimental datasets. Nucleic Acids Res. 2019:47:D607-D613. https://doi.org/10.1093/nar/gky1131 
16. Sakalihasan N, Michel J-B, Katsargyris A, Kuivaniemi H, Defraigne J-O, Nchimi A, et al. Abdominal aortic aneurysms. Nat Rev Dis Primers. 2018;4:34. https://doi.org/10.1038/s41572-018-0030-7.

17. Anagnostakos J, Lal BK. Abdominal aortic aneurysms. Prog Cardiovasc Dis. 2021;65:34-43. https://doi.org/10.1016/.jpcad.2021.03.009.

18. Golledge J. Abdominal aortic aneurysm: update on pathogenesis and medical treatments. Nat Rev Cardiol. 2019;16:225-42. https://doi.org/10 1038/s41569-018-0114-9.

19. Radonic T, de Witte P, Groenink M, de Waard V, Lutter R, van Eijk M, et al. Inflammation Aggravates Disease Severity in Marfan Syndrome Patients. PLoS ONE. 2012;7: e32963. https://doi.org/10.1371/journal.pone.0032963.

20. Hinterseher I, Tromp G, Kuivaniemi H. Genes and Abdominal Aortic Aneurysm. Ann Vasc Surg. 2011;25:388-412. https://doi.org/10.1016/j. avsg.2010.09.004.

21. A IJpma, L te Riet, KM van de Luijtgaarden, P M van Heijningen, J Burger, D Majoor-Krakauer, et al. Inflammation and TGF- $\beta$ Signaling Differ between Abdominal Aneurysms and Occlusive Disease. J Cardiovasc Dev Dis. 2019;6:38. https://doi.org/10.3390/jcdd6040038

22. Guedj K, Khallou-Laschet J, Clement M, Morvan M, Delbosc S, Gaston A-T, et al. Inflammatory Micro-Environmental Cues of Human Atherothrombotic Arteries Confer to Vascular Smooth Muscle Cells the Capacity to Trigger Lymphoid Neogenesis. PLoS ONE. 2014;9: e1 16295. https://doi. org/10.1371/journal.pone.0116295.

23. Kitchen CM, Cowan SL, Long X, Miano JM. Expression and Promoter Analysis of a Highly Restricted Integrin Alpha Gene in Vascular Smooth Muscle. Gene. 2013;513:82-9. https://doi.org/10.1016/j.gene.2012.10.073,

24. Chen J, Maltby KM, Miano JM. A novel retinoid-response gene set in vascular smooth muscle cells. Biochem Biophys Res Commun. 2001;281:475-82. https://doi.org/10.1006/bbrc.2001.4362.

25. Wulf-Johansson H, Lock Johansson S, Schlosser A, Anne Trommelholt Holm, Lars Melholt Rasmussen, Hans Mickley, et al. Localization of microfibrillar-associated protein 4 (MFAP4) in human tissues: clinical evaluation of serum MFAP4 and its association with various cardiovascuIar conditions. PLoS One. 2013;8:e82243. https://doi.org/10.1371/journal. pone. 0082243

26. Lindholt JS, Madsen M, Kirketerp-Møller KL, Schlosser A, Kristensen KL, Andersen $C B$, et al. High plasma microfibrillar-associated protein 4 is associated with reduced surgical repair in abdominal aortic aneurysms. J Vasc Surg. 2020;71:1921-9. https://doi.org/10.1016/j.jvs.2019.08.253.

27. Guo DC, Pannu H, Tran-Fadulu V, Papke CL, Yu RK, Avidan N, et al. Mutations in smooth muscle a-actin (ACTA2) lead to thoracic aortic aneurysms and dissections. Nat Genet. 2007;39:1488-93. https://doi.org/10.1038/ng. 2007.6.

28. Yokoyama U, Arakawa N, Ishiwata R, Yasuda S, Minami T, Goda M, Uchida $\mathrm{K}$, Suzuki S, et al. Proteomic analysis of aortic smooth muscle cell secretions reveals an association of myosin heavy chain 11 with abdominal aortic aneurysm. Am J Physiol Heart Circ Physiol. 2018;315:H1012-8. https://doi.org/10.1152/ajpheart.00329.2018.

29. Licht AH, Nübel T, Feldner A, Jurisch-Yaksi N, Marcello M, Demicheva E, et al. Junb regulates arterial contraction capacity, cellular contractility, and motility via its target Myl9 in mice. J Clin Invest. 2010;120:2307-18. https://doi.org/10.1172/JCl41749.

30. Nogi M, Satoh K, Sunamura S, Kikuchi N, Satoh T, Kurosawa R, et al. SmgGDS Prevents Thoracic Aortic Aneurysm Formation and Rupture by Phenotypic Preservation of Aortic Smooth Muscle Cells. Circulation. 2018;138:2413-33. https://doi.org/10.1161/CIRCULATIONAHA.118. 035648.

31. Lampson M Fan, Gillian Douglas, Jennifer K Bendall, Eileen McNeill, Mark J Crabtree, Ashley B Hale, et al. Endothelial cell-specific reactive oxygen species production increases susceptibility to aortic dissection. Circulation. 2014;129:2661-72. https://doi.org/10.1161/CIRCULATIONAHA.113. 005062

32. Fan LM, Douglas G, Bendall JK, Lampson M Fan, Gillian Douglas, Jennifer K Bendall, et al. Endothelial cell-specific reactive oxygen species production increases susceptibility to aortic dissection. Circulation.2014;129:2661-72. https://doi.org/10.1161/CIRCULATIONAHA.113.005062

33. Shalata A, Mahroom M, Milewicz DM, Limin G, Kassum F, Badarna K, et al. Fatal thoracic aortic aneurysm and dissection in a large family with a novel MYLK gene mutation: delineation of the clinical phenotype. Orphanet J Rare Dis. 2018;13:41. https://doi.org/10.1186/ s13023-018-0769-7.

34. Nanda V, Miano JM. Leiomodin 1, a New Serum Response Factor-dependent Target Gene Expressed Preferentially in Differentiated Smooth Muscle Cells. J Biol Chem. 2012;287:2459-67. https://doi.org/10.1074/jbc.M111. 302224.

35. Mayanagi T, Sobue K. Diversification of caldesmon-linked actin cytoskeleton in cell motility. Cell Adh Migr. 2011;5:150-9. https://doi.org/10.4161/ cam.5.2.14398.

36. Goikuria H, Del Mar Freijo M, Manrique RV, Sastre M, Elizagaray E, Lorenzo A, et al. Characterization of Carotid Smooth Muscle Cells during Phenotypic Transition. Cells. 2018;7:23. https://doi.org/10.3390/cells7030023

37. Nanda V, Wang T, Pjanic M, Liu B, Nguyen T, Matic LP, et al. Functional regulatory mechanism of smooth muscle cell-restricted LMOD1 coronary artery disease locus. PLoS Genet. 2018;14:e1007755. https://doi.org/10. 1371/journal.pgen.1007755

38. Zhang J, Zhang J, Shouping Xu, Zhang X, Wang P, Hao Wu, et al. HypoxiaInduced TPM2 Methylation is Associated with Chemoresistance and Poor Prognosis in Breast Cancer. Cell Physiol Biochem. 2018;45:692-705. https://doi.org/10.1159/000487162.

39. Meng L-B, Shan M-J, Qiu Y, Qi R, Ze-Mou Yu, Guo P, et al. TPM2 as a potential predictive biomarker for atherosclerosis. Aging (Albany NY). 2019;11:6960-82. https://doi.org/10.18632/aging.102231.

\section{Publisher's Note}

Springer Nature remains neutral with regard to jurisdictional claims in published maps and institutional affiliations.

Ready to submit your research? Choose BMC and benefit from:

- fast, convenient online submission

- thorough peer review by experienced researchers in your field

- rapid publication on acceptance

- support for research data, including large and complex data types

- gold Open Access which fosters wider collaboration and increased citations

- maximum visibility for your research: over $100 \mathrm{M}$ website views per year

At BMC, research is always in progress.

Learn more biomedcentral.com/submissions 